\title{
Hyperactivation and Acrosome Reaction In Vitro in Spermatozoa Ejaculated by Cryptorchid Dogs after Orchiopexy
}

\author{
Eiichi KAWAKAMI, Harumichi NAITOH, Michiru OGASAWARA, Miyuki TAMURA, Junichi HASEGAWA, \\ Toshihiko TSUTSUI, and Akira OGASA \\ Department of Reproduction, Nippon Veterinary and Animal Science University, 1-7-1 Kyonan-cho, Musashino-shi, Tokyo 180, Japan
}

(Received 10 January 1990/Accepted 8 January 1991)

\begin{abstract}
Orchiopexy of the cryptorchid (CR) testis and castration of the scrotal testis were performed in three unilaterally $\mathrm{CR}$ beagles at six months of age. Induction rates for ejaculated sperm hyperactivation (HA) and the acrosome reaction (AR) in vitro in these orchiopexied dogs were compared with five those in normal beagles one year later. Canine spermatozoa were incubated for $9 \mathrm{hr}$ at $38^{\circ} \mathrm{C}$ under $5 \% \mathrm{CO}_{2}$ in air in canine capacitation medium at a concentration of $30 \times 10^{6} \mathrm{sperm} / \mathrm{m} l$. HA was observed using high-speed videomicrography. The AR spermatozoa were evaluated by the triple stain technique. As a result, there was no significant difference between 'the CR dogs after orchiopexy' (CDO) and the normal dogs (ND) with respect to sperm motility just after ejaculation. However, sperm motility of CDO decreased markedly during incubation. There was a significant difference in sperm motility between CDO (Mean $\pm \mathrm{SD} ; 47 \pm 12 \%)$ and ND $(80 \pm 9 \%)$ after three hours of incubation $(\mathrm{p}<0.01)$. No significant difference was observed between CDO and ND with respect to the HA rate of motile spermatozoa throughout the incubation period. The peak of HA rate was found in both CDO $(58 \pm 5 \%)$ and ND $(61 \pm 16 \%)$ after seven hours of incubation. The AR rate

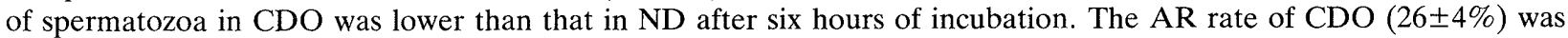
significantly lower than ND $(46 \pm 5 \%)$ after eight hours of incubation $(\mathrm{p}<0.01)$. It is assumed that there might be relation between a rapid decrease of motility and low AR rate in spermatozoa of CDO during incubation.- KEY wORDS: capacitation, cryptorchidism, dog, spermatozoa.
\end{abstract}

The authors have previously reported that orchiopexy in cryptorchid $(\mathrm{CR})$ dogs gives rise to the appearance of spermatozoa in the orchiopexied testes [14]. There is not much difference between spermatozoa produced in the orchiopexied testes and those in normal dog's testes with respect to motility and viability just after semen collection. However, the fertilizing capacity of orchiopexied dogs's spermatozoa was revealed to be quite poor [15]. The mammalian spermatozoa must undergo a process called capacitation consisting of hyperactivation (HA) and acrosome reaction (AR) in order to penetrate eggs $[3,4,12,25]$. In many species including the dog, sperm $\mathrm{HA}$ and $\mathrm{AR}$ can be observed by means of experiments in vivo and in vitro $[6,8,9,11,17,18,21,24]$. In the present experiment, induction rates for HA and AR in vitro of spermatozoa ejaculated by 'the CR dogs after orchiopexy' (CDO) and the normal dogs (ND) were investigated in order to elucidate the cause of the poor fertilizing ability of spermatozoa of the former.

MATERIALS AND METHODS

Animals and semen collection: Three unilaterally inguinal CR beagles, six months of age, were subjected to orchiopexy of the CR testis and castration of the scrotal testis by the method previously described [14]. Semen samples were collected weekly by digital manipulation and their quality was tested by the methods previously described [13]. The spermatozoa were incubated in vitro one year later when the characteristics of the ejaculate in $\mathrm{CDO}$ became stable. Five control beagles of the same age as $\mathrm{CDO}$ were examined in the same manner.

Sperm incubation: The medium used for incubation of spermatozoa was canine capacitation medium (CCM) solution ( $\mathrm{pH} \mathrm{7.8,} \mathrm{osmotic} \mathrm{pressure}$ $300 \mathrm{~m} \mathrm{Osm} / \mathrm{kg}$ ) developed by Mahi and Yanagimachi [18], containing bovine serum albumin 2 $\mathrm{mg} / \mathrm{m} l$, penicillin $\mathrm{G} 100 \mathrm{U} / \mathrm{m} l$, and streptomycin 0.05 $\mathrm{mg} / \mathrm{ml}$. A $3 \mathrm{~m} l$ of CCM medium was placed in glass test tube $(100 \times 13 \mathrm{~mm})$ covered with aluminium cap. Semen characteristics were tested immediately after collection and spermatozoa were washed by centrifugation ( $200 \mathrm{~g}, 5 \mathrm{~min})$ with CCM medium. The washed spermatozoa were resuspended in the same medium to give a concentration of $30 \times 10^{6}$ sperm $/ \mathrm{m} l$ and were incubated for $9 \mathrm{hr}$ at $38^{\circ} \mathrm{C}$ under 


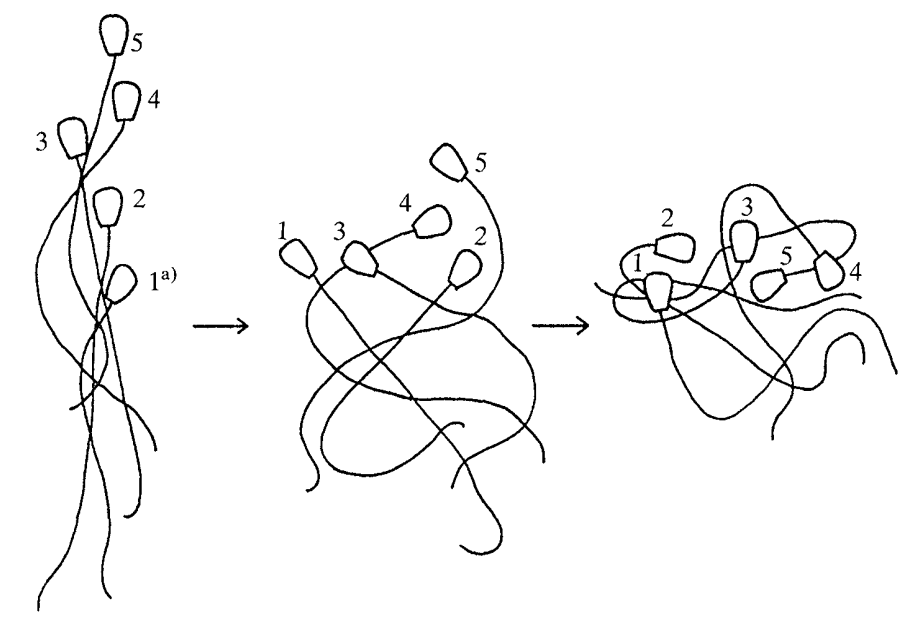
(A) Non-hyperactivated
(B) Traditional
(C) Hyperactivated

Fig. 1. Motility patterns of incubated dog spermatozoa.

a) These figures show successive positions of a spermatozoon traced at 1/50 sec. intervals.

$5 \% \mathrm{CO}_{2}$ in air.

Observation of $H A$ and $A R$ : Sperm HA and AR were checked after $0,1,3,5,6,7,8$ and 9 hours of incubation. Sperm movement patterns were recorded by high-speed videomicrography (Nac Co., Ltd., Tokyo, Japan) at 1/50 sec. intervals. Movement pattern A in Fig. 1 was exhibited by all motile spermatozoa just after semen collection. Spermatozoa which showed movement pattern $\mathrm{C}$ increased gradually in number throughout the incubation period. According to other reports $[5,19,26]$, hyperactivated sperm are considered to exhibit movement pattern C. HA rate of sperm was analyzed by examination of 100 motile sperm which were recorded on a videotape. Live, acrosomereacted sperm were identified using the triple stain technique described by Talbot and Chacon [22]. Sperm with white heads, except for brown postacrosomal segments, were classified as live and acrosome-reacted and the rate of these sperm was expressed as AR rate.

\section{RESULTS}

Semen was collected four times every week from three CDO and five ND. There was no difference in sperm motility between CDO (Mean \pm SD; $86.7 \pm$ $4.7 \%)$ and ND $(96.2 \pm 1.5 \%)$.

Changes in sperm motility during incubation in these CDO and ND are shown in Fig. 2. In ND the mean motility of the incubated spermatozoa more

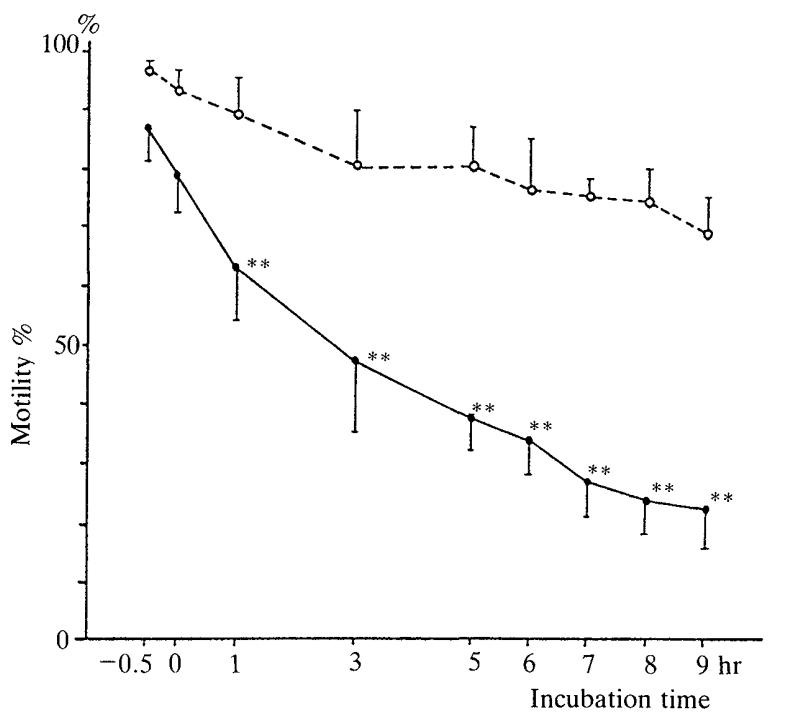

Fig. 2. The mean ( \pm S.D.) rates of motility in spermatozoa during incubation in 3 orchiopexied (- - ) and 5 normal dogs $(\cdots \bigcirc-\cdots)$.

${ }^{* *}$ Significant difference from spermatozoa of the normal dogs during incubation $(\mathrm{P}<0.01)$.

than $70 \%$ was maintained until eight hours of incubation. In CDO, however, the mean motility decreased markedly with increasing incubation time and there was a significant difference in the mean motility between incubated CDO and ND spermatozoa after one hour of incubation $(p<0.01)$. The sperm motilities of CDO and ND after three hours of incubation were $47 \pm 12 \%$ and $80 \pm 9 \%$, respectively. The $\mathrm{HA}$ rates of motile spermatozoa are 


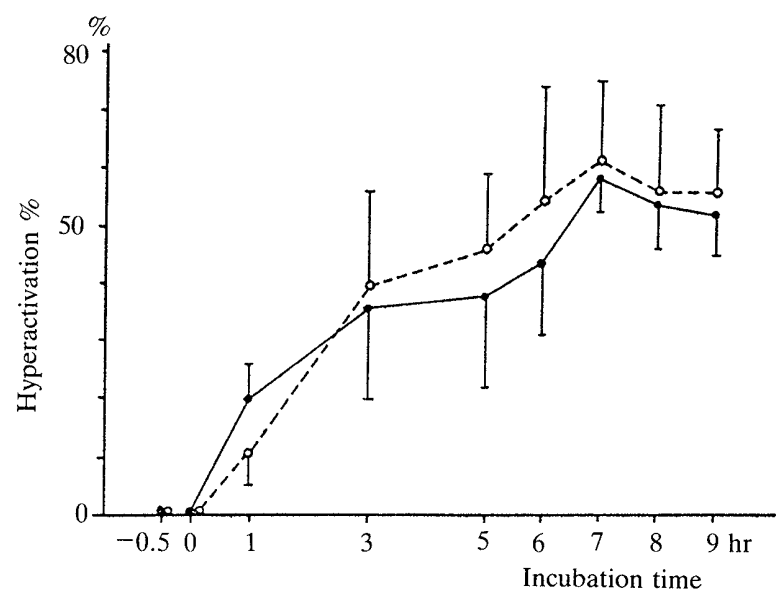

Fig. 3. The mean ( \pm S.D.) rates of hyperactivation in spermatozoa during incubation in 3 orchiopexied $(--)$ and 5 normal dogs $(\cdots \bigcirc \cdots)$.

shown in Fig. 3. Changes in the HA rates of incubated spermatozoa in CDO and ND showed similar patterns, and their HA rates were both increased after one hour of incubation. The HA rates of $\mathrm{CDO}$ and ND peaked after seven hours of incubation at $58 \pm 5 \%$ and $61 \pm 16 \%$, respectively.

The AR rates of the incubated CDO and ND spermatozoa are shown in Fig. 4. In spermatozoa ejaculated by $\mathrm{CDO}$ the increase in the $\mathrm{AR}$ rate disappeared after six hours of incubation and there was a significant difference in the rate between $\mathrm{CDO}$ $(26 \pm 4 \%)$ and ND $(46 \pm 5 \%)$ after eight hours of incubation $(\mathrm{p}<0.05)$.

\section{DISCUSSION}

Mahi and Yanagimachi (1978) reported that the active motility and AR rates of ND spermatozoa are $80-90 \%$ and $46 \%$, respectively, after seven hours of incubation [18]. In this study, those of ND spermatozoa were $75 \pm 2 \%$ and $40 \pm 11 \%$, respectively, after seven hours of incubation. The results of the present study are in well accordance with theirs.

It is known that enzymes such as ATPase and minerals such as $\mathrm{Ca}^{2+}$ are important to the movement and energy metabolism of spermatozoa $[1,2$, $7,23]$. Nagano reported that sperm of the artificial crytorchid rat has abnormal ultrastructure of the tail [20]. If spermatozoa of CDO have abnormal metabolic systems and/or abnormal ultrastructures in the tail, these sperm motility and viability may remarkably decrease during incubation. It is supposed that such a characteristics of CDO spermatozoa may

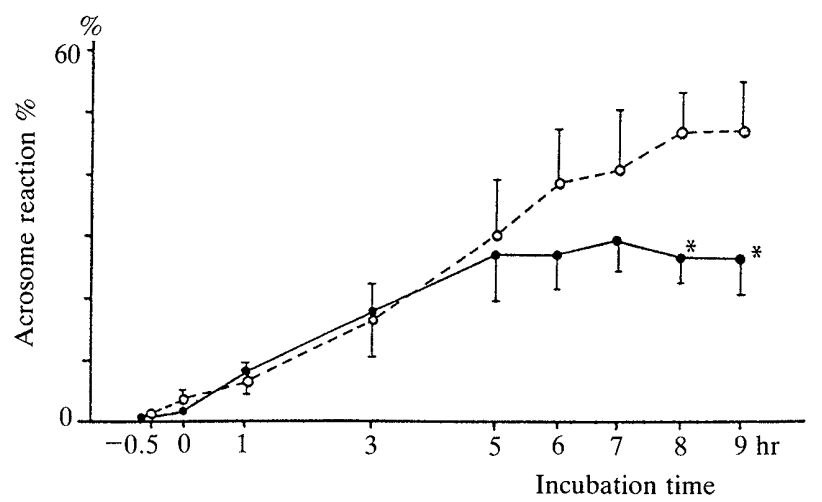

Fig. 4. The mean ( \pm S.D.) rates of acrosome reaction in spermatozoa during incubation in 3 orchiopexied ( - ) and 5 normal dogs $(\cdots \bigcirc \cdots)$.

"Significant difference from spermatozoa of the normal dogs during incubation $(\mathrm{P}<0.05)$.

cause the poor fertility. It seems, therefore, necessary to study the ultrastructure of spermatozoa produced by orchiopexied testes in the future.

As far as the HA rate of motile spermatozoa is concerned, there is not much difference between $\mathrm{CDO}$ and ND. It is reported that decapacitation factors are components of seminal plasma $[10,16]$. This study revealed that HA of spermatozoa of CDO and ND was similarly induced because of removal of decapacitation factors by washing of their spermatozoa with medium. It is assumed that there might be a relation between a rapid decrease of motility and low AR rate in spermatozoa of CDO during incubation.

REFERENCES

1. Amelar, R. D., Dubin, L., and Schoenfeld, C. 1980. Sperm motility. Fertil. Steril. 34: 197-215.

2. Ashraf, M., Peterson, R. N., and Russell, L. D. 1984. Characterization of $\left(\mathrm{Ca}^{2+}+\mathrm{Mg}^{2+}\right)$ adenosine triphosphatase activity and calcium transport in boar sperm plasma membrane vesicles and their relation to phosphorylation of plasma membrane proteins. Biol. Reprod. 31: 1061-1071.

3. Barros, C. and Garavagno, A. 1970. Capacitation of hamster spermatozoa with blood sera. J. Reprod. Fertil. 22: 381-384.

4. Bedford, J. M. 1968. Ultrastructural changes in the sperm head during fertilization in the rabbit. Am. J. Anat. 123: 329-358.

5. Burkman, L. J. 1984. Characterization of hyperactivated motility by human spermatozoa during capacitation: Comparison of fertile and oligozoospermic sperm populations. Arch. Androl. 13: 153-165.

6. Byrd, W. 1981. In vitro capacitation and the chemically induced acrosome reaction in bovine spermatozoa. J. Exp. Zool. 215: 35-46.

7. Clarke, G. N., Clarke, F. M., and Wilson, S. 1982. Actin in 
human spermatozoa. Biol. Reprod. 26: 319-327.

8. Cummins, J. M. 1982. Hyperactivated motility patterns of ram spermatozoa recovered from the oviducts of mated ewe. Gamete Res. 6: 53-63.

9. Fleming, A. D. and Yanagimachi, R. 1982. Fertile life of acrosome reacted guinea pig spermatozoa. J. Exp. Zool. 220: 109-115.

10. Fraser, L. R. 1984. Mouse sperm capacitation in vitro involves loss of a surface-associated inhibitory component. J. Reprod. Fertil. 72: 373-384.

11. Goodrowe, K. L., Wall, R. J., O'Brien, S. J., Schmidt, P. M., and Wildt, D. W. 1988. Developmental competence of domestic cat follicular oocytes after fertilization in vitro. Biol. Reprod. 39: 355-372.

12. Hamner, C. E., Jennings, J. J., and Sojka, N. J. 1970. Cat (Felis Catus L.) spermatozoa require capacitation. J. Reprod. Fertil. 23: 477-480.

13. Kawakami, E., Tsutsui, T., Yamada, Y., Ogasa, A., and Yamauchi, M. 1988. Spermatogenic function in cryptorchid dogs after orchiopexy. Jpn. J. Vet. Sci. 50: 227-235.

14. Kawakami, E., Tsutsui, T., Yamada, Y., Ogasa, A., and Yamauchi, M. 1988. Spermatogenic function and fertility in unilateral cryptorchid dogs after orchiopexy and contralateral castration. Jpn. J. Vet. Sci. 50: 754-762.

15. Kawakami, E., Tsutsui, T., Yamada, Y., and Yamauchi, M. 1984. Cryptorchidism in the dog: Occurrence of crytorchidism and semen quality in the cryptorchid dog. Jpn. J. Vet. Sci. 46: 349-356.

16. Lamirande, E. D., Belles-Isles, M., and Gagnon, C. 1984. Characteristics of a seminal plasma inhibitor of sperm motility. Ann. New York Acad. Sci. 438: 125-131.
17. Mahi, C. A. and Yanagimachi, R. 1976. Maturation and sperm penetration of canine ovarian oocytes in vitro. $J$. Exp. Zool. 196: 189-195.

18. Mahi, C. A. and Yanagimachi, R. 1978. Capacitation, acrosome reaction, and egg penetration by canine spermatozoa in a simple defined medium. Gamete Res. 1: 101-109.

19. Morales, P., Overstreet, J. W., and Katz, D. F. 1988. Changes in human sperm motion during capacitation in vitro. J. Reprod. Fertil. 83: 119-128.

20. Nagano, T. 1963. Fine structural changes in the flagellum of the spermatid in experimental cryptorchidism of the rat. $J$. Cell Biol. 18: 337-344.

21. Shalgi, R. and Phillips, D. M. 1988. Motility of rat spermatozoa at the site of fertilization. Biol. Reprod. 39: 1207-1213.

22. Talbot, P. and Chacon, R. S. 1981. Triple-stain technique for evaluating normal acrosome reactions of human sperm. J. Exp. Zool. 215: 201-208.

23. Tash, J. S. and Means, A. R. 1983. Cyclic adenosine $3^{\prime}, 5^{\prime}$ mono-phosphate, calcium and protein phosphorylation in flagellar motility. Biol. Reprod. 28: 75-104.

24. Varner, D. D., Ward, C. R., Storey, B. T., and Kenney, R. M. 1987. Induction and characterization of acrosome reaction in equine spermatozoa. Am. J. Vet. Res. 48: 1383-1389.

25. Yanagimachi, R. 1970. In vitro capacitation of golden hamster spermatozoa by homologous and heterologous blood sera. Biol. Reprod. 3: 147-153.

26. Yanagimachi, R. 1970. The movement of golden hamster spermatozoa before and after capacitation. J. Reprod. Fertil. 23: 193-196. 